\title{
Laparoscopic management of early primary peritoneal pregnancy: a case report
}

\author{
Hwa Seon Koo', Ju Youn Bae', Inn Soo Kang', Mi Kyoung Koong', Hye Ok Kim', Sun Hwa Cha', Min Hye Choi', Ji Young Kim², \\ Kwang Moon Yang ${ }^{1}$
}

'Department of Obstetrics \& Gynecology, ${ }^{2}$ Department of Pathology, Cheil General Hospital and Women's Healthcare Center, Kwandong University College of Medicine, Seoul, Korea

Peritoneal pregnancy is an implantation in the peritoneal cavity exclusive of tubal, ovarian, or intra-ligamentary implantations. This is a rare obstetric complication with high maternal mortality and even higher perinatal mortality, and secondary type was most common. Risk factors for peritoneal pregnancy are previous history of extrauterine pregnancy or tubal surgery pelvic post-inflammatory status or presence of an intrauterine device. As it is a life-threatening condition, expectant management carries a risk of sudden life-threatening intra-abdominal bleeding and a generally poor fetal prognosis. So, when it is recognized, immediate termination of pregnancy is usually recommended. Early diagnosis of peritoneal pregnancy is difficult, but is important by their life threatening progress course to patients. Recently, we experienced primary peritoneal pregnancy which meets both the original and modified criteria. In this paper, we reported the case of early diagnosed and successfully treated peritoneal pregnancy despite of their diagnosis was incidentally.

Keywords: Primary Peritoneal Pregnancy; Ectopic Pregnancy; Laparoscopic Management; Human

\section{Introduction}

The blastocyst normally implants in the endometrial lining of the uterine cavity. Implantation anywhere else is considered an ectopic pregnancy. Nearly $95 \%$ of ectopic pregnancies are implanted in one of the segments of the fallopian tubes. The remaining $5 \%$ implant in the ovary, peritoneal cavity, or within the cervix [1]. Strictly defined, peritoneal pregnancy is an implantation in the peritoneal cavity exclusive of tubal, ovarian, or intra-ligamentary implantations [2].

Although primary peritoneal pregnancy is defined as an ectopic

Received: May 7, 2011 · Revised: May 31, 2011 · Accepted: Jun 3, 2011

Corresponding author: Kwang Moon Yang

Department of Obstetrics and Gynecology, Cheil General Hospital and Women's Healthcare Center, Kwandong University College of Medicine, 1-19 Mukjeongdong, Jung-gu, Seoul 100-380, Korea

Tel: +82-2-2000-4728 Fax:+82-2-2000-7790 E-mail:km1yang@naver.com

This is an Open Access article distributed under the terms of the Creative Commons Attribution Non-Commercial License (http://creativecommons.org/licenses/by-nc/3.0/) which permits unrestricted non-commercial use, distribution, and reproduction in any medium, provided the original work is properly cited. pregnancy that implants primarily on a peritoneal surface, most abdominal pregnancies are thought to fellow early tubal rupture or abortion [3]. In 1942, Studdiford [4] reported a well-documented case and established criteria for defining problem cases. This criteria was modified by Friedrich and Rankin [5] in 1968.

As it is a life-threatening condition, expectant management carries a risk of sudden life-threatening intra-abdominal bleeding and a generally poor fetal prognosis. Therefore, when it is recognized, immediate termination of pregnancy is usually recommended [6]. Although early diagnosis of peritoneal pregnancy is difficult, it is important due to the life threatening nature of this complication.

Recently, we experienced early diagnosis and successfully treated primary peritoneal pregnancy and report it here with a brief review of the literature.

\section{Case report}

A 32-year-old gravid 1, parity 0 woman presented with a history of 

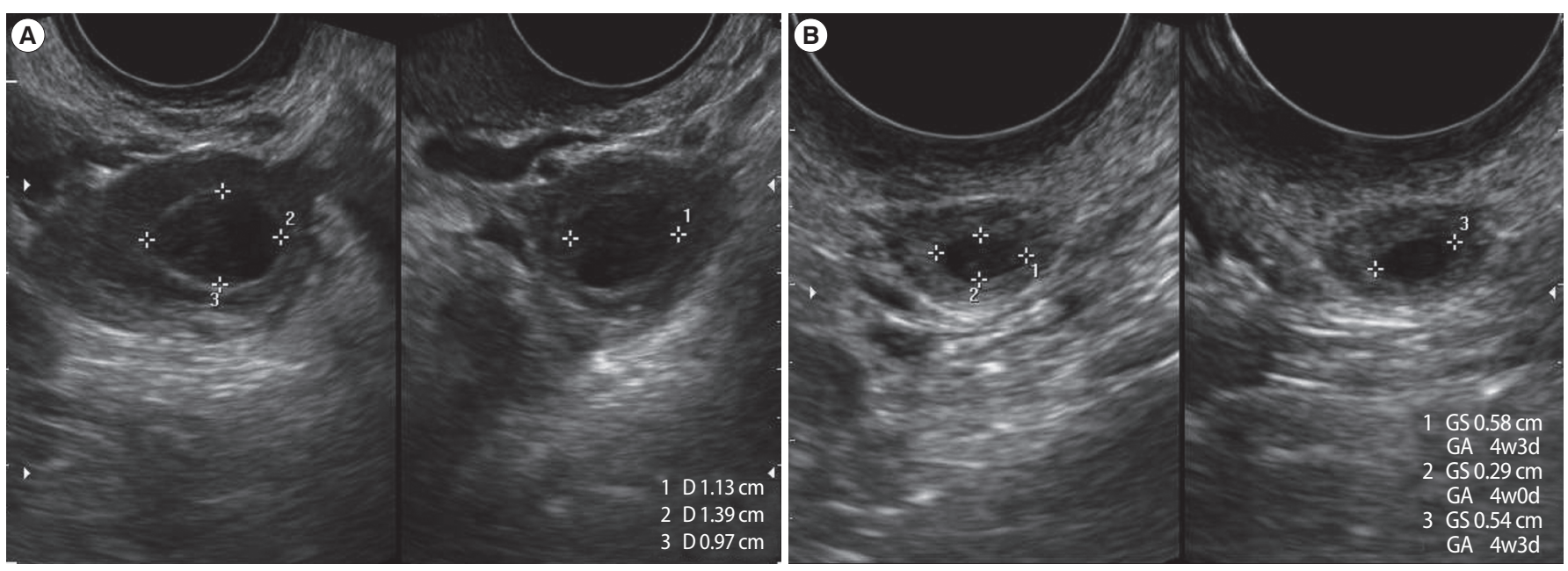

Figure 1.Transvaginal ultrasound findings at amenorrhea $5^{+1}$ weeks which suggested extra-uterine pregnancy in the left adnexa. $(A) A$ hyperechoic mass was detected in the lateral aspect of the left ovary. (B) A gestational sac-like structure was detected in the left ovarian hyper-echoic mass.

secondary infertility for 7 months. Her chief complaint was wanting a baby. The patient's menstrual periods were irregular with a cycle length of 25-33 days. Her obstetrical history included one missed abortion about 7 months prior to the visit. Five times of timed intercourse were carried out for pregnancy success at another infertility clinic before visiting our infertility center. On physical and transvaginal ultrasound examination, which was done at the first visit, nothing unusual was found in the patient.

At the 18th menstrual day, a 16-mm growing follicle was diagnosed in the left ovary by ultrasound examination and we advised her to carry out timed intercourse at the 20th and 22nd menstrual days. At the 31st menstrual day (amenorrhea $4^{+3}$ weeks), she visited the clinic for a pregnancy test and had the level of serum $\beta$-hCG checked, which was found to be $406.5 \mathrm{mlU} / \mathrm{mL}$. At the 36th menstrual day (amenorrhea $5^{+1}$ weeks), serum $\beta$-hCG was increased to $2,053.0 \mathrm{mlU} / \mathrm{mL}$, but no gestational sac was detected within the uterus by transvaginal ultrasound. A $1.1 \times 1.0 \times 0.7 \mathrm{~cm}$ sized hyperechoic mass containing a $0.6 \times 0.5 \times 0.3 \mathrm{~cm}$ sized gestational sac-like structure was detected in the lateral aspect of the left ovary by ultrasonographic examination (Figure 1). A diagnosis of ectopic pregnancy in left tube was made. Two treatment options--medical and surgical--with detailed information on their advantages, disadvantages, and side effects were offered. Surgical removal of the ectopic mass was chosen. The patient was hospitalized and laparoscopic surgery was performed on the day of diagnosis.

On laparoscopic examination, the uterus was slightly enlarged with a small myoma on the uterine fundus. The right ovary, bilateral salpingeal tubes, omentum, and pelvic peritoneum were grossly normal without any suspected lesion of ectopic pregnancy. However, an approximately $1 \times 2-\mathrm{cm}$ cystic mass on the left ovary and an approxi-

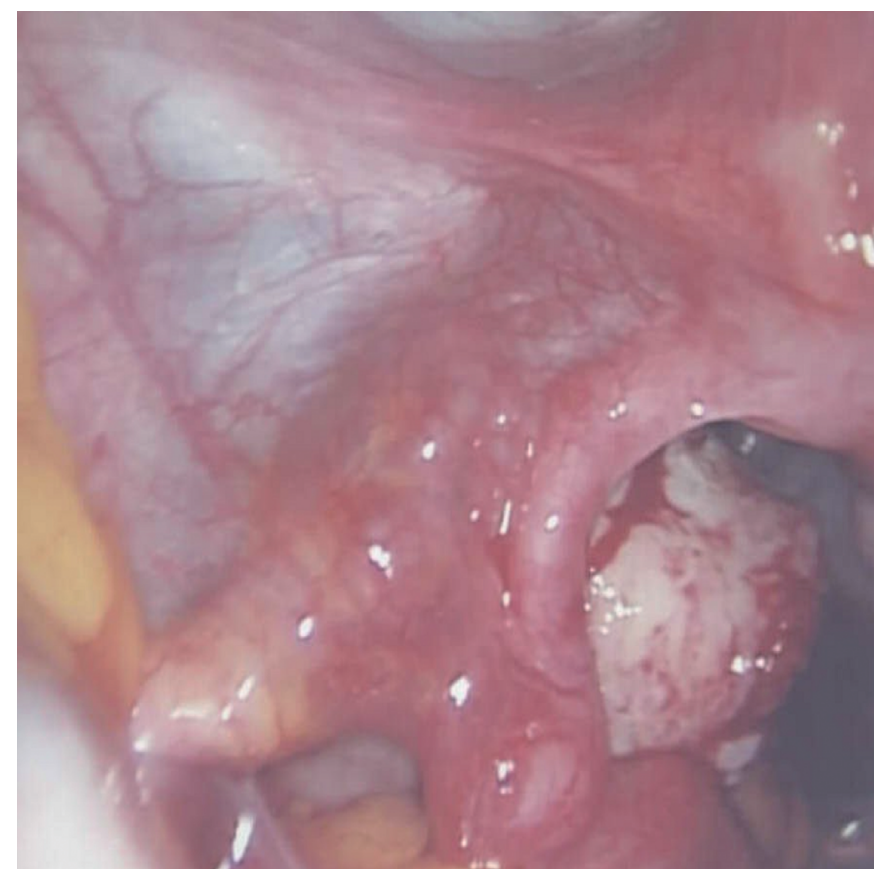

Figure 2. Laparoscopic findings of initial operation. A reddish and edematous mass on the left infundibulopelvic ligament was detected on laparoscopic examination.

mately $2 \times 4-\mathrm{cm}$ reddish and edematous mass on the left infundibulopelvic ligament were detected on laparoscopic examination (Figure 2). Those masses were excised and sent to pathologists to rule out ectopic pregnancy. After that, dilatation and curettage of the endometrium was performed to rule out complicated early intrauterine pregnancy. The cyst masses on the left ovary and left infundibulopelvic ligament were described, respectively, as a lutenized follicular cyst and an unusual ectopic adrenal gland with no clinical significance by 
pathologist reports 1 week after the operation. The endometrial sample was reported as gestational endometrium without trophoblastic tissue (Figure 3).

One day after operation, laboratory tests showed $\mathrm{Hb} / \mathrm{Hct}$ was 11.9 $\mathrm{g} / \mathrm{dL} / 34.5 \%$ and serum $\beta$-hCG level had increased to $2,107 \mathrm{mlU} / \mathrm{mL}$ post-operatively compared to a pre-operative level of $2,053 \mathrm{mlU} / \mathrm{mL}$.
A sustained pregnancy at a site other than the uterus, bilateral salpingeal tubes, or bilateral ovary was suspected. For the cessation of sustained abnormal pregnancy, single intra-muscular injection of 75 mg methotrexate (MTX) (50 mg/1 m² of body surface area) was done. The patients was discharged without any discomfort and planned to follow up 5 days after the MTX injection.
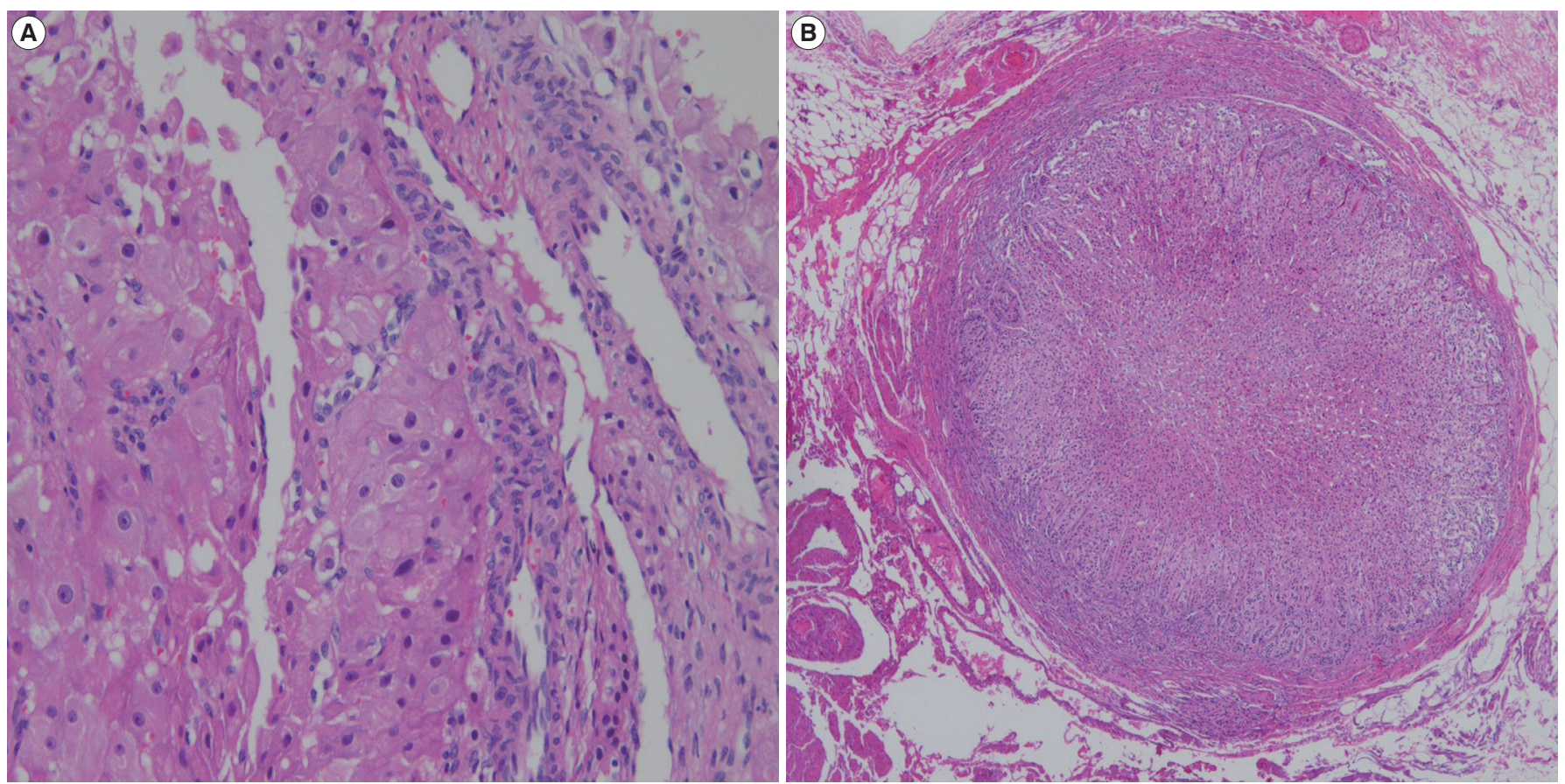

Figure 3. Pathologic findings of excised masses from the initial operation. (A) Cystic mass on the left ovary: lutenized cells with abundant eosinophilic to clear cytoplasm lined cyst (left side) and lie within its fibrous wall (right side) (H\&E stain, $\times 200)$. (B) Reddish nodular mass on the infundibulopelvic ligament: three cortical layers resembling adrenal glands without medullary tissue (H\&E stain, $\times 40)$.
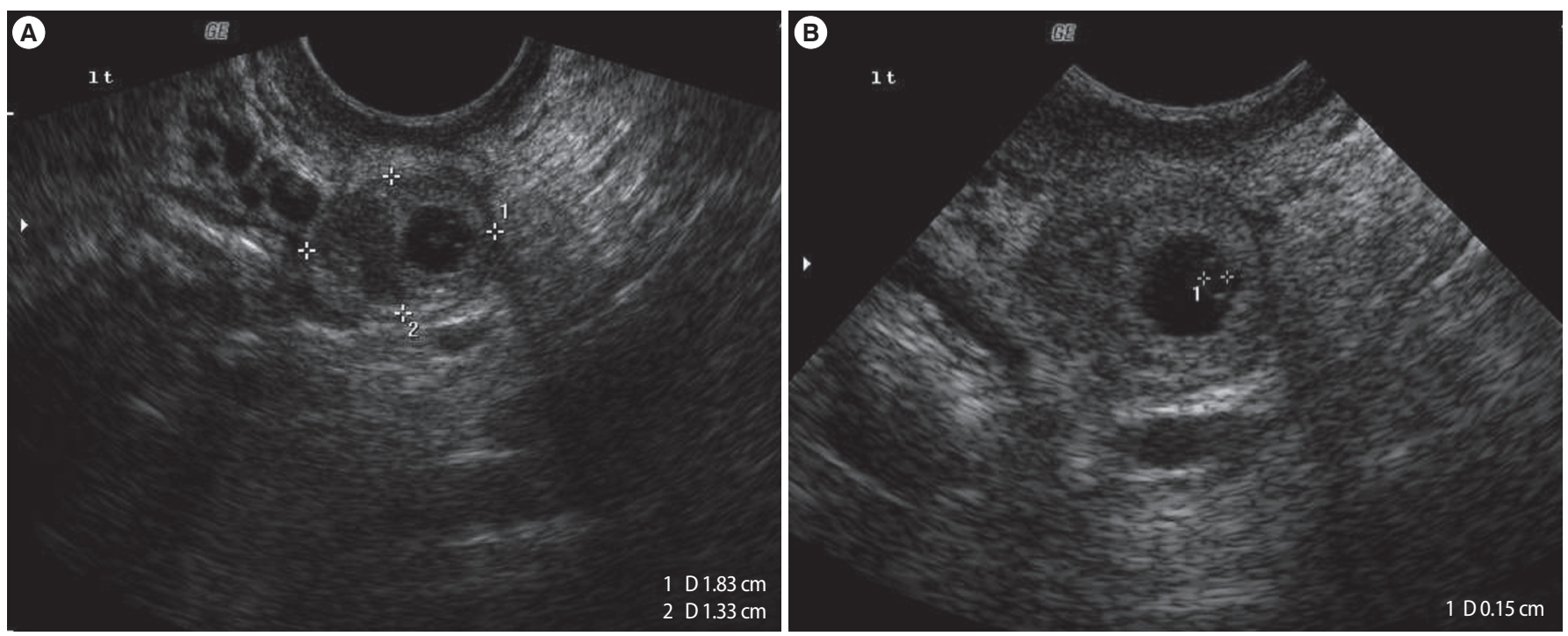

Figure 4. Transvaginal ultrasound findings at 5 days after MTX injection, which corresponded to post-operative 6 days and amenorrhea $6^{+0}$ weeks. (A) An echogenic mass was noted in the left adnexa lateral to the left ovary. (B) An inner gestational sac-like structure and 0.2-cm sized yolk sac-like structure with fetal heart movement. 
At 5 days after MTX injection, which corresponded to post-operative 6 days and amenorrhea $6^{+0}$ weeks, serum $\beta$-HCG was checked

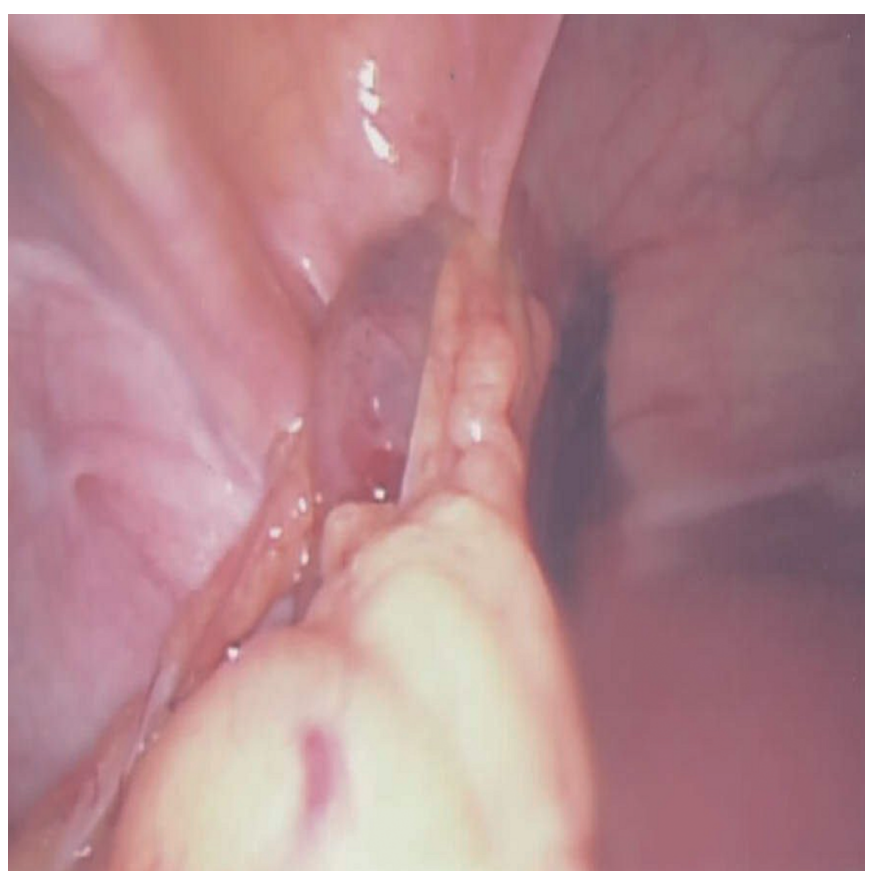

Figure 5. Laparoscopic findings of re-operation. A reddish mass was detected at the pelvic peritoneum, which was located in the cul-desac area adjacent to the lateral aspect of the left utero-sacral ligament. again and the level had increased to $2,955 \mathrm{mlU} / \mathrm{mL}$. A $1.8 \times 1.7 \times 1.3$ $\mathrm{cm}$ echogenic mass that contained an inner gestational sac-like structure and $0.2 \mathrm{~cm}$ yolk sac-like structure with fetal heart movement in the left adnexal area lateral to the left ovary was detected on transvaginal ultrasonogram (Figure 4). Late implanted extra-uterine pregnancy was suspected. Re-operation by laparoscopic surgery was chosen and performed immediately.

On laparoscopic examination, an approximately $2 \times 2 \mathrm{~cm}$ reddish mass was detected in the pelvic peritoneum, located in the cul-desac area adjacent to the lateral aspect of the left utero-sacral ligament (Figure 5). Also, the left fallopian tube was swollen and had an edematous appearance. The pelvic peritoneal mass was excised widely with the adjacent peritoneum. A left salpingectomy was performed to rule out left tubal pregnancy. Those samples were sent to the pathology department for confirmation of excised extrauterine pregnancy. The pathologist reported that villous trophoblasts were present on the pelvic peritoneal mass, which indicated ectopic pregnancy. The left tube that appeared edematous was revealed to have inflammatory changes due to the operation done 6 days earlier (Figure 6).

One day after re-operation, the laboratory test for $\mathrm{Hb} / \mathrm{Hct}$ was 10.9 $\mathrm{g} / \mathrm{dL} / 33 \%$ and serum $\beta$-hCG had decreased to $505 \mathrm{mlU} / \mathrm{mL}$. The patient was discharged, and follow up 7 days after re-operation was planned. Seven days after re-operation, the patient was evaluated on an outpatient basis. The serum $\beta$-HCG had decreased to $33.8 \mathrm{mIU} /$
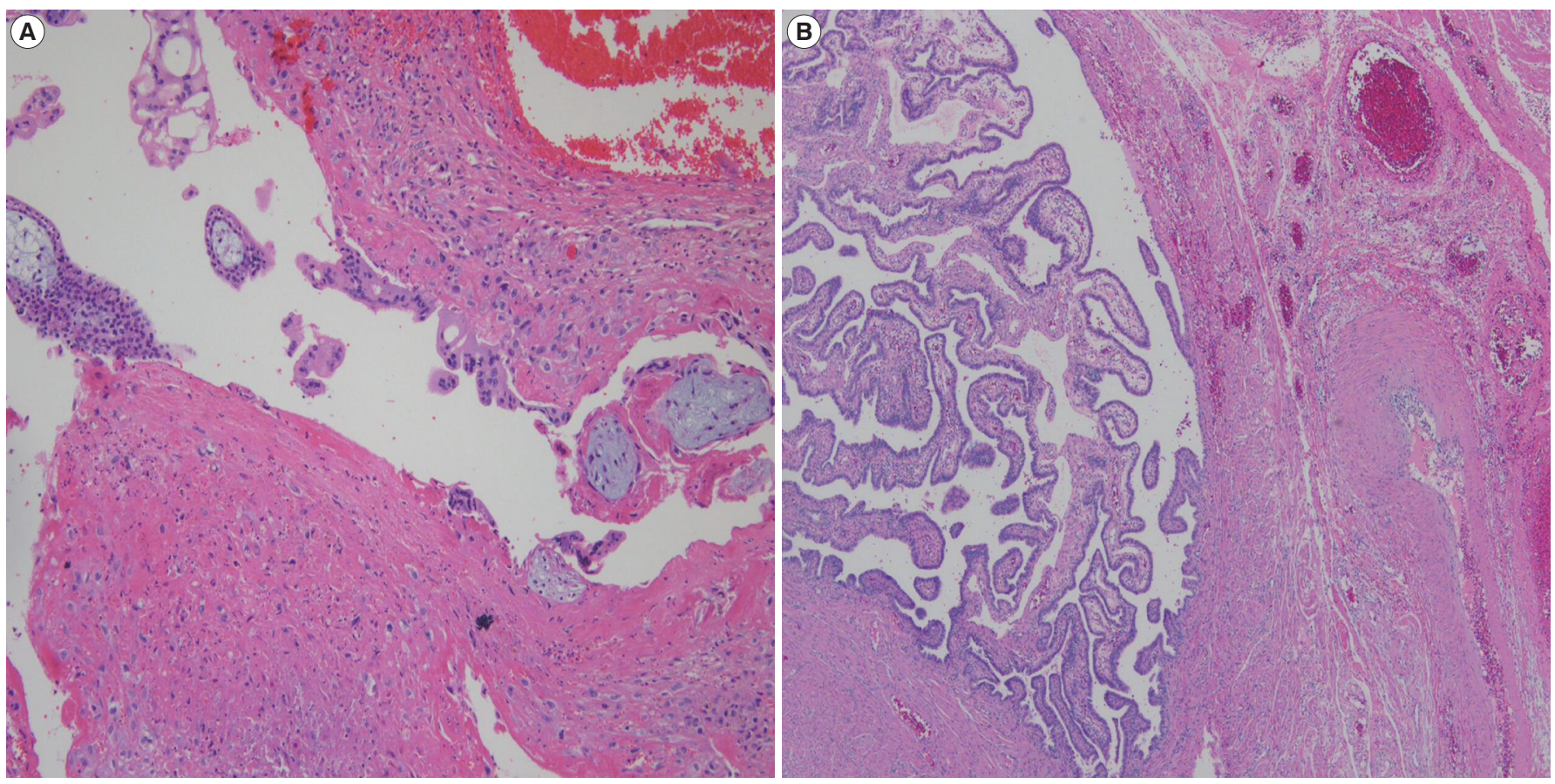

Figure 6. Pathologic findings of excised pelvic peritoneal mass and salpingectomy specimen at re-operation. (A) Pelvic peritoneal mass: degenerated villous trophoblasts, consistent with ectopic pregnancy (H\&E stain, $\times 100)$. (B) Salpingectomy specimen: infiltrated chronic inflammatory cells and hemorrhagic congestion (H\&E stain, $\times 100)$. 
$\mathrm{mL}$, and the patient's postoperative recovery was uneventful with no bothersome symptoms or signs.

\section{Discussion}

Primary peritoneal pregnancy was defined as implantation of the fertilized egg on the pelvic peritoneum. Primary peritoneal pregnancy is a clinically accepted rare entity whose pathogenesis remains controversial. In 1942, Studdiford [4] reported a case of an early ruptured pregnancy on the posterior aspect of the uterus, which, based on its relationship to the interstitial portion of the tube and to the peritoneum, was accepted as the first reported primary peritoneal nidation. After that, primary peritoneal pregnancy was clinically distinguished from secondary peritoneal pregnancy by Studdiford's established criteria [4]. Studdiford's criteria include the following:

1) Normal tubes and ovaries with no evidence of recent or remote injury,

2) An absence of any evidence of a utero-peritoneal fistula,

3) The presence of a pregnancy related exclusively to the peritoneal surface and early enough to eliminate the possibility of secondary implantation following a primary nidation in the tube.

In a review of the literature, Friedrich and Rankin found only 24 cases of first trimester pregnancies where the implantation site was solely on the peritoneal surface [4]. They proposed modifying Studdiford's criteria to limit acceptable cases such that tubal damage should be clearly evident and the placental site would be distinct. Friedrich and Rankin's modifications are as follows:

1) The presence of a pregnancy of less than 12 weeks' histological gestational age whose trophoblastic attachments are related solely to a peritoneal surface

2) Grossly normal tubes and ovaries

3) The absence of utero-peritoneal fistula

In contrast to primary peritoneal pregnancy, secondary peritoneal pregnancy, which is a condition where the embryo or fetus continues to grow in the abdominal cavity after its expulsion from the fallopian tube or other seat of its primary development, occurs in 1 in 10,000 live births [7].

Risk factors for peritoneal pregnancy are the same as for ectopic pregnancy, such as previous history of extrauterine pregnancy or tubal surgery (including sterilizations), pelvic post-inflammatory status (adhesions proved by coelioscopy) or presence of an intrauterine device [1].

The diagnosis of peritoneal pregnancy is complex. Ultrasound, when coupled with clinical evaluation, has approximately a 50 percent success rate in the diagnosis [8]. The guidelines for the use of ultrasound to diagnose abdominal pregnancy require: 1 ) demonstration of a fetus in a gestational sac outside the uterus, or the depiction of an ab- dominal or pelvic mass identifiable as the uterus separate from the fetus; 2 ) failure to see a uterine wall between the fetus and urinary bladder; 3 ) recognition of a close approximation of the fetus to the material abdominal wall; and 4) localization of the placenta outside the confines of the uterine cavity. However, the reported diagnostic error rates in different series have ranged from 50 to 90\% [9]. An magnetic resonance imaging scan can also be used to confirm the diagnosis of abdominal pregnancy. Laboratory tests, such as abnormally increasing human chorionic gonadotropin, are not sufficiently reliable on their own to make a diagnosis, nor are signs and symptoms such as abdominal pain and tenderness, persistent transverse or oblique lie, or palpable fetal parts [8].

Peritoneal pregnancy poses an equally serious threat to the survival of the mother and the fetus, especially in the case of diagnosis delayed up to the second trimester of pregnancy. Maternal mortality ranges between 0 and $30 \%$ [10]. This is principally because of the risk of massive hemorrhage from incomplete or entire placental separation. The placenta can be attached to the uterine wall, bowel, mesentery, liver, spleen, bladder, and ligaments, which can separate at any time during pregnancy, leading to heavy blood loss. The fetal outcome tends to be poorer than the mother's perinatal mortality, ranging between 40 and 95\% [11]. Fetal abnormalities are also high, with a number of congenital malformations being common. However, with advanced pregnancy and if the fetus is surrounded by a normal volume of amniotic fluid, fetal outcome tends to be better [10].

Factors such as maternal hemodynamic status, fetal congenital abnormality, fetal viability, gestational age at presentation, and the availability of neonatal facilities should be considered before management of peritoneal pregnancy [12]. If the fetus is dead, surgical intervention is generally indicated owing to the risk of infection and disseminated intravascular coagulation. If the fetus is alive, laparotomy should be performed, regardless of gestational age or fetal condition. The reason is mainly based on the unpredictability of placental separation and consequential massive haemorrhage [13]. Pre-operative systemic methotrexate with subsequent laparotomy for removal of the fetus and placenta may minimize potential blood loss, and may be a reasonable approach in the care of a patient with an abdominal pregnancy with placental implantation to abdominal viscera and blood vessels. However, this treatment option could be considered in the management of this potentially life-threatening condition, but more reports and experience are required [6].

In our case, the evidence of extra-uterine pregnancy was not detected in the pelvic peritoneum during the first laparoscopic surgery but was detected in the second operation. The only evidence of pregnancy after the first operation was the sustained level of serum $\beta$ hCG, which was not decreased by MTX injection. We assumed there were two reasons for the delayed diagnosis of abdominal pregnancy: 
1) a delayed type of peritoneal implantation, 2) slow growth of conceptual tissues by their poor nutritional environment in the peritoneum. Hence, the second operation seemed inevitable for the diagnosis of early peritoneal pregnancy described in our case.

Early diagnosis of peritoneal pregnancy is difficult, but is important due to the life threatening nature of the condition. We reported a case of peritoneal pregnancy early diagnosed and successfully treated despite incidental diagnosis.

\section{Conflict of interest}

No potential conflict of interest relevant to this article was reported.

\section{References}

1. Stucki D, Buss J. The ectopic pregnancy, a diagnostic and therapeutic challenge. J Med Life 2008;1:40-8.

2. Worley KC, Hnat MD, Cunningham FG. Advanced extrauterine pregnancy: diagnostic and therapeutic challenges. Am J Obstet Gynecol 2008;198:297.e1-7.

3. Cunningham F, Leveno K, Bloom S, Hauth J, Rouse D, Spong C. Williams obstetrics. 23rd ed. New York, NY: McGraw-Hill Professional; 2010.
4. Studdiford WE. Primary peritoneal pregnancy. Am J Obstet Gynecol 1942;44:487-91.

5. Friedrich EG Jr, Rankin CA Jr. Primary pelvic peritoneal pregnancy. Obstet Gynecol 1968;31:649-53.

6. Gupta P, Sehgal A, Huria A, Mehra R. Secondary abdominal pregnancy and its associated diagnostic and operative dilemma: three case reports. J Med Case Reports 2009;3:7382.

7. Atrash HK, Friede A, Hogue CJ. Abdominal pregnancy in the United States: frequency and maternal mortality. Obstet Gynecol 1987;69:333-7.

8. Costa SD, Presley J, Bastert G. Advanced abdominal pregnancy. Obstet Gynecol Surv 1991;46:515-25.

9. Allibone GW, Fagan CJ, Porter SC. The sonographic features of intra-abdominal pregnancy. J Clin Ultrasound 1981;9:383-7.

10. Martin JN Jr, McCaul JF 4th. Emergent management of abdominal pregnancy. Clin Obstet Gynecol 1990;33:438-47.

11. Ang LP, Tan AC, Yeo SH. Abdominal pregnancy: a case report and literature review. Singapore Med J 2000;41:454-7.

12. Shafi SM, Malla MA, Salaam PA, Kirmani OS. Abdominal pregnancy as a cause of hemoperitoneum. J Emerg Trauma Shock 2009;2:196-8.

13. Maas DA, Slabber CF. Diagnosis and treatment of advanced extra-uterine pregnancy. S Afr Med J 1975;49:2007-10. 